\title{
Left Paraduodenal Hernia Incidentally Diagnosed during Operation for Transverse Colon Cancer
}

\author{
Tomoko Seya ${ }^{1,2}$, Noritake Tanaka ${ }^{1,2}$, Kimiyoshi Yokoi ${ }^{1.2}$, \\ Susumu Okada ${ }^{3}$, Yoshiharu Oaki ${ }^{4}$ and Eiji Uchida ${ }^{1}$ \\ ${ }^{1}$ Surgery for Organ Function and Biological Regulation, \\ Graduate School of Medicine, Nippon Medical School \\ ${ }^{2}$ Department of Surgery, Nippon Medical School Chiba Hokusoh Hospital \\ ${ }^{3}$ Department of Radiology, Nippon Medical School Chiba Hokusoh Hospital \\ ${ }^{4}$ Department of Pathology, Nippon Medical School Chiba Hokusoh Hospital
}

\begin{abstract}
We report the case of a patient with paraduodenal hernia diagnosed incidentally during an operation for transverse colon cancer. The patient was a 77-year-old woman who complained of dizziness. Laboratory data revealed no abnormal findings except slight anemia. Barium enema and colonoscopic examination revealed an irregular surfaced mass, about $5.0 \mathrm{~cm}$ in size, located near the flexure of the spleen of the transverse colon. A biopsy of the mass was performed, and a moderately differentiated adenocarcinoma was diagnosed. In April 2009, following the diagnosis of transverse colon cancer, laparotomy was performed, which revealed that a few loops of the jejunum were herniated through the orifice into the space posterior to the transverse mesocolon. Moreover, the jejunal loops were located right between a shifted left branch of the middle colic artery and ascending left colic artery. There were no ischemic changes in the jejunum. These findings were consistent with a left paraduodenal hernia associated with transverse colon cancer. The scheduled left hemicolectomy was performed in addition to a radical operation of the left paraduodenal hernia. The abdominal computed tomography (CT) images were reviewed postoperatively. The scan projection radiogram obtained by $\mathrm{CT}$ revealed a packing of jejunal loops in the middle of the abdomen. Abdominal CT revealed ascending left colic artery at the left edge of a packing of jejunal loops. The patient was discharged from our hospital 14 days after the surgery without any complications. Left paraduodenal hernias are rare and constitute less than $0.4 \%$ of all intestinal obstructions. Retrospectively reviewed, the preoperative $\mathrm{CT}$ is suggestive. In addition to the packing of jejunal loops in the middle of the abdomen, ascending left colic artery was clearly observed at the left edge of the packing of jejunal loops, which indicates left paraduodenal hernia.

(J Nippon Med Sch 2010; 77: 111-114)
\end{abstract}

Key words: left paraduodenal hernia, transverse colon cancer, computed tomography (CT)

Correspondence to Tomoko Seya, MD, PhD, Department of Surgery, Nippon Medical School Chiba Hokusoh Hospital, 1715 Kamagari, Inba-mura, Inba-gun, Chiba 270-1694, Japan

E-mail: seya@nms.ac.jp

Journal Website (http://www.nms.ac.jp/jnms/) 


\section{Introduction}

Internal abdominal hernias are rare, accounting for $0.9 \%$ of all intestinal obstructions ${ }^{1}$. Paraduodenal hernias constitute $53 \%$ of all internal abdominal hernias ${ }^{2}$. The symptoms of paraduodenal hernia vary from asymptomatic to severe septic shock depending upon the degree of small bowel obstruction $^{3}$. Here, we present a case of left paraduodenal hernia complicated with transverse colon cancer, which was incidentally diagnosed during an operation for transverse colon cancer.

\section{Case Report}

A 77-year-old woman was referred to our hospital with a diagnosis of transverse colon cancer. Her chief complaint was dizziness. She had no family history except that her two brothers were treated for prostate cancer. Laboratory data revealed slight anemia and the patient's serum carcinoembryonic antigen and CA19-9 levels were normal. Physical examination revealed no abnormal findings. Barium enema and colonoscopic examination revealed an irregular surfaced mass, about $5.0 \mathrm{~cm}$ in size, located near the flexure of the spleen of the transverse colon. A biopsy of the mass was performed, and a moderately differentiated adenocarcinoma was diagnosed. In April 2009, following the diagnosis of transverse colon cancer, laparotomy was performed, and it revealed that a few loops of the jejunum were herniated through the orifice into the space posterior to the transverse mesocolon (Fig. 1a). Moreover, the jejunal loops were located right between a shifted left branch of the middle colic artery and ascending left colic artery. There were neither volvulus nor ischemic changes in the jejunum. These findings were consistent with the left paraduodenal hernia associated with transverse colon cancer. The scheduled left hemicolectomy was performed in addition to a radical operation of the
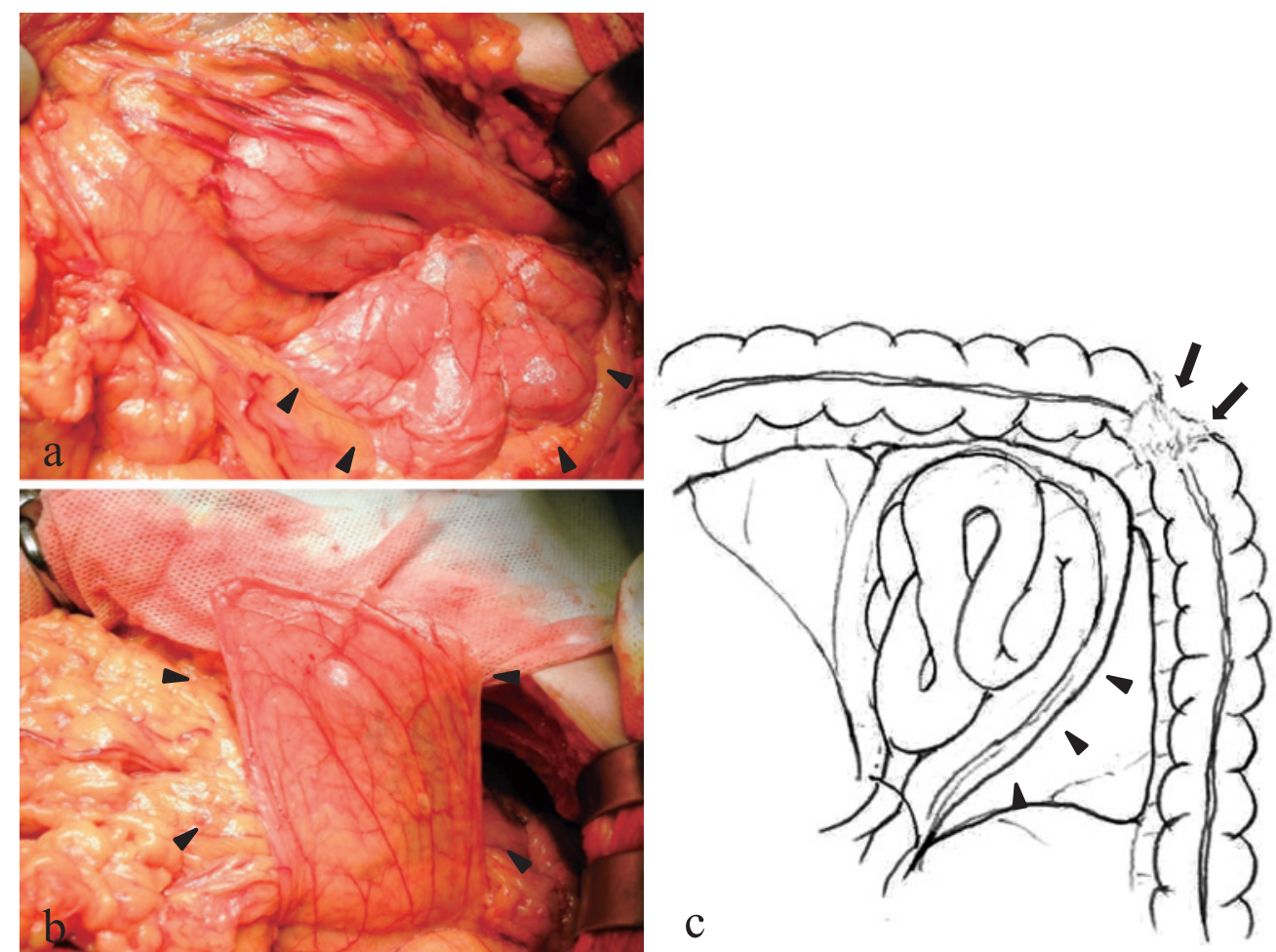

Fig. 1 a: Operative view: jejunal loops (arrowheads) encapsulated in a peritoneal sac were observed. b: Operative view: a nonviable sac (arrowheads) was observed after reducing incarcerated jejunum. c: Diagram of the left paraduodenal hernia. The small bowel loops herniated via the fossa of Landzert into left portion of the transverse mesocolon. Ascending left colic artery was the left margin of the neck of the sac (arrowheads). Transverse colon cancer was located in the splenic flexure (arrows). 

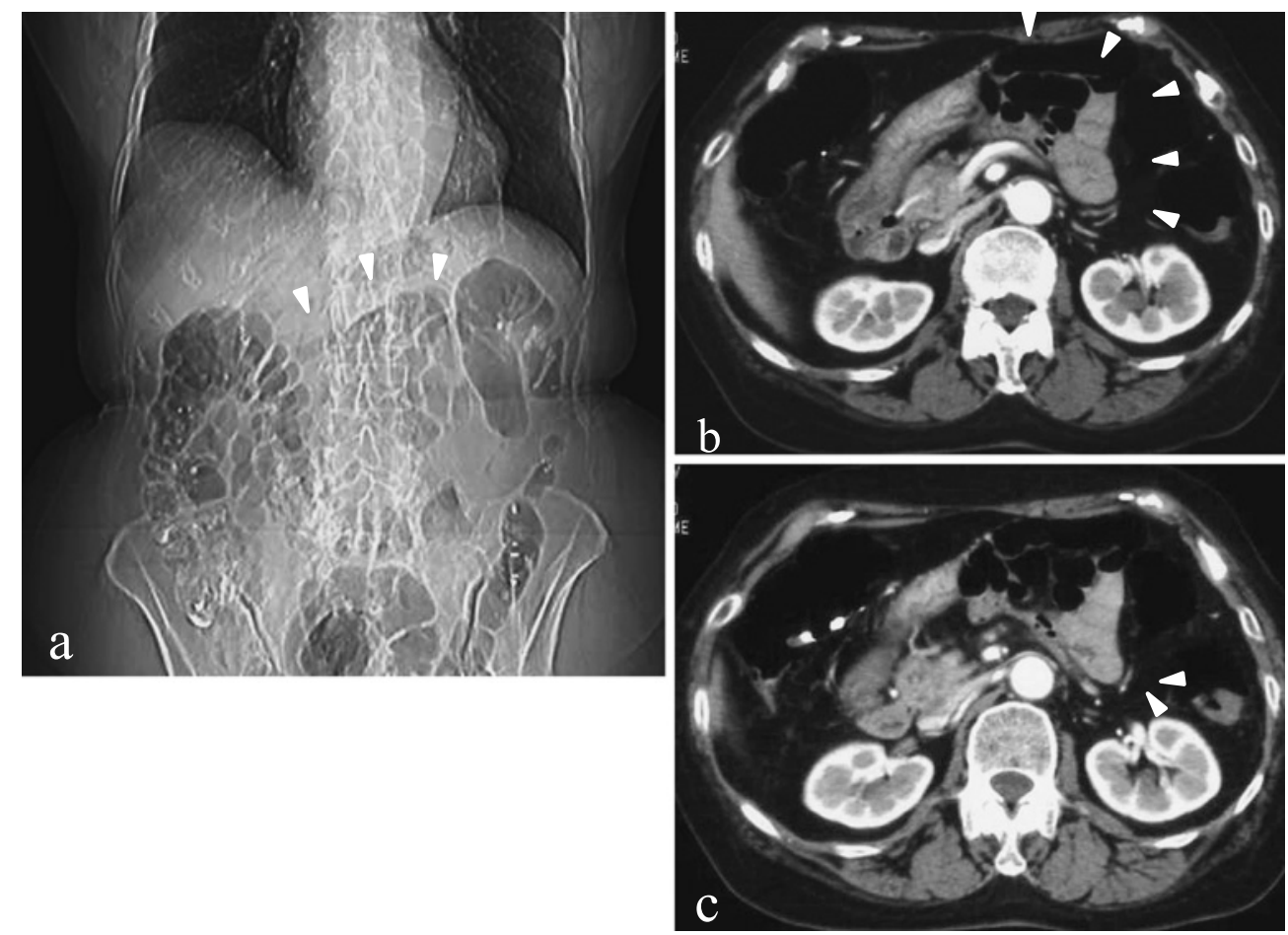

Fig. 2 a: The scan projection radiogram obtained by CT revealed a packing of small bowel loops (arrowheads) in the middle of the abdomen. b: A packing of small bowel loops (arrowheads) in the middle of the abdomen was revealed in the CT images. c: Ascending left colic artery (arrowheads) was revealed at the left edge of a packing of small bowel loops.

left paraduodenal hernia; i.e., after reducing the jejunum, a nonviable sac was excised (Fig. 1b) and the hernia orifice was sutured and closed. The diagram of the hernia is shown in Figure 1c. The abdominal computed tomography (CT) films were reviewed postoperatively. The scan projection radiogram obtained by $\mathrm{CT}$ revealed a packing of jejunal loops in the middle of the abdomen (Fig. 2a), which were also revealed in the $\mathrm{CT}$ images (Fig. 2b). The ascending left colic artery was observed at the left edge of a packing of jejunal loops (Fig. 2c). The patient was discharged from our hospital 14 days after the surgery without any complications.

\section{Discussion}

Paraduodenal hernias are rare congenital errors in the embryologic rotation of the midgut and variations in peritoneal fixation and vascular folds. They are classified according to the site of the fossa developing into the sac of the hernia. Left paraduodenal hernia is three times more common than right paraduodenal hernia'. It develops through the fossa of Landzert at the left of the fourth portion of the duodenum, because the inferior mesenteric vein runs along and above the lateral side of the fossa, which raises the peritoneal fold. Left paraduodenal hernia often makes a diagnostic confusion because of herniated loops present at a distance ${ }^{2}$. We must clearly understand the location of the hernia orifice and the position of the inferior mesenteric artery and ascending left colic artery. The left paraduodenal hernia contains only a few loops of the jejunum and sometimes is reduced spontaneously ${ }^{2.5}$. The main symptoms vary depending on the degree of bowel obstruction, ranging from mild gastrointestinal symptoms to septic shock due to incarcerated bowel necrosis ${ }^{3}$. The present patient was asymptomatic and diagnosed incidentally during an operation for transverse colon cancer. Although a few loops of the jejunum were herniated through the orifice into the space posterior to the transverse mesocolon, there was no jejunal obstruction leading to ischemic 
changes as shown in the operation. This explains the absence of symptoms. However, if she had had recurrent abdominal discomfort before the operation, we would prejudge that it was due to the transverse colon cancer. On the basis of nonspecific clinical manifestations, a preoperative diagnosis of paraduodenal hernia is difficult, particularly in cases accompanied by other obstructive diseases. It is important for clinicians to be aware of the existence of paraduodenal hernia in patients who experience intermittent abdominal pain.

After the operation, we reviewed the radiologic findings carefully. To establish the diagnosis of paraduodenal hernia, radiologic studies are essential. Generally, plain film of the abdomen may demonstrate a distended fluid-filled stomach or dilated loops of small bowel in an ovoid mass lateral from the midline ${ }^{6}$. In the present patient, the scan projection radiogram obtained by $\mathrm{CT}$ revealed a packing of jejunal loops in the middle of the abdomen. CT revealed ascending left colic artery at the left edge of the packing of jejunal loops. This finding coincided with the operative finding that indicated left paraduodenal hernia. CT is now one of the most effective methods for the diagnosis of paraduodenal hernia, in addition to upper gastrointestinal series, ultrasonography, and celiac angiography ${ }^{1,6}$.

After a diagnosis of paraduodenal hernia is made, the treatment is surgery. The basic principles of surgery for paraduodenal hernias are reduction of hernia contents and repair of the defect by either closure or widening of the hernia orifice ${ }^{7}$. In our patient, after reducing the herniated jejunum, the nonviable sac was excised and the hernia orifice was sutured and closed. Recently, the laparoscopic management of paraduodenal hernias has been increasingly carried out ${ }^{8.9}$. There is a report that, in the case of massive incarceration and malrotation of the small intestine, a suitable operative field could not be obtained by laparoscopic techniques. Therefore, the laparoscopic approach is indicated in nonobstructive left paraduodenal hernias at present ${ }^{8}$. Regrettably, in the present patient, the laparoscopic approach was not carried out because an advanced transverse colon cancer was present. For patients diagnosed preoperatively as having paraduodenal hernia, a minimally invasive approach may be feasible. In the future, laparoscopic surgery will be widely and safely applied to many types of internal hernia.

We report the case of a left paraduodenal hernia as a coincidental finding in a patient who underwent laparotomy for transverse colon cancer. The present patient was not accompanied by bowel obstruction as shown in the operation; a careful reading of radiologic results should be important for patients with intermittent abdominal pain. On the basis of a clear understanding of paraduodenal hernia, appropriate surgical treatment is necessary.

\section{References}

1. Khan MA, Lo AY, Vande Maele DM: Paraduodenal hernia. Am Surg 1998; 64: 1218-1222.

2. Meyers MA: Dynamic radiology of the abdomen. Normal and Pathologic anatomy. Chapter 16, 2000; pp 711-748, Springer, Berlin, Heidelberg, New York.

3. Yoo HY, Mergelas J, Seibert DG: Paraduodenal hernia. A treatable cause of upper gastrointestinal tract symptoms. J Clin Gastroenterol 2000; 31: 226229 .

4. Zimmerman LM, Laufman H: Intraabdominal hernias due to developmental and rotational abnormalies. Ann Surg 1953; 138: 82-91.

5. Manji R, Warnock GL: Left paraduodenal hernia: an unusual cause of small-bowel obstruction. Can J Surg 2001; 44: 455-457.

6. Hirasaki S, Koide N, Shima Y, et al.: Unusual variant of left paraduodenal hernia herniated into mesocolic fossa leading to jejunal stranglation. J Gastroenterol 1998; 33: 734-738.

7. Bartlett MK, Wang C, Williams WH: The surgical management of paraduodenal hernia. Ann Surg 1968; 168: 249-254.

8. Uematsu T, Kitamura $\mathrm{H}$, Iwase $\mathrm{M}$, et al.: Laparoscopic repair of paraduodenal hernia. Surg Endosc 1998; 12: 50-52.

9. Uchiyama S, Imamura $\mathrm{N}$, Hidaka $\mathrm{H}$, et al.: An unusual variant of a left paraduodenal hernia diagnosed and treated by laparoscopic surgery: report of a case. Surg Today 2009; 39: 533-535.

(Received, September 15, 2009)

(Accepted, October 27, 2009) 